cemoti $\begin{aligned} & \text { Cahiers d'études sur la Méditerranée } \\ & \text { orientale et le monde turco-iranien }\end{aligned}$

$20 \mid 1995$

Médias d'Iran et d'Asie Centrale

\title{
Les médias au Tadjikistan
}

Mina RAD

\section{(2) OpenEdition}

Journals

Édition électronique

URL : http://journals.openedition.org/cemoti/1673

DOI : 10.4000/cemoti. 1673

ISSN : $1777-5396$

Éditeur

AFEMOTI

Édition imprimée

Date de publication : 1 juin 1995

ISSN : 0764-9878

Référence électronique

Mina RAD, «Les médias au Tadjikistan », Cahiers d'études sur la Méditerranée orientale et le monde turcoiranien [En ligne], 20 | 1995, mis en ligne le 13 mai 2006, consulté le 08 septembre 2020. URL : http:// journals.openedition.org/cemoti/1673; DOI : https://doi.org/10.4000/cemoti.1673

Ce document a été généré automatiquement le 8 septembre 2020

Tous droits réservés 


\title{
Les médias au Tadjikistan
}

\author{
Mina RAD
}

1 Le Tadjikistan est un exemple type de pays de l'ex-Union Soviétique qui accède à l'indépendance et bascule dans la guerre civile. La situation des médias en est une très bonne illustration. Le vent de liberté qui a soufflé sur l'Union Soviétique gorbatchévienne a entraîné la libération des médias; après 70 ans de communisme et de domination totalitaire, la presse devenait le principal lieu de la liberté d'expression mais, rapidement contrôlée par les différents groupes se disputant le pouvoir, elle s'est fait le porte-parole de la guerre des idées, les plumes précédant les fusils.

2 C'est donc dans un contexte de tension sociale et de confusion politique que nous aurons à étudier l'évolution des médias tadjiks.

Le poids du totalitarisme

3 Pendant 70 ans, les journaux étaient tous publiés sous le contrôle du régime communiste. Dans toutes les républiques soviétiques, la presse était étroitement contrôlée par le pouvoir central. Toutes les publications étaient centralisées dans la capitale, concentrées dans un seul bâtiment où se trouvait, en général, la seule imprimerie pour tous les journaux du pays.

4 L'architecture et l'atmosphère du bâtiment de la presse sont aussi symboliques. Le bâtiment s'élève sur plusieurs étages avec de longs couloirs où règne le climat pesant d'une presse muselée. Le trajet que doit suivre le moindre article est clairement fixé. Il est particulièrement significatif du système de contrôle de la presse. De la salle de rédaction, l'article doit monter au sommet de la hiérarchie du journal, au dernier étage du bâtiment, où se trouve le bureau du "directeur général", puis passer par l'étage en dessous, au "bureau de la censure", avant de redescendre à l'imprimerie.

5 Un journaliste tadjik (qui a tenu à garder l'anonymat) explique: "Chaque article, avant d'être envoyé à l'imprimerie, doit être lu d'abord par le directeur, puis par le bureau de la censure. Si cet article est un peu "critique" ou bien "suspect", il n'aura pas l'autorisation de continuer son chemin vers l'imprimerie".

6 Même pendant la brève période où le Tadjikistan a connu une grande liberté, ces règles furent maintenues, témoignant ainsi des limites du changement. Le rédacteur en chef du journal tadjik indépendant, Tcheraq-e Rouz, le confirme: "Les jours où il y avait un 
article trop critique à l'égard du régime, notre journal n'était pas publié, le gouvernement invoquait alors le trop traditionnel prétexte des "problèmes techniques"".

$7 \quad$ En ce qui concerne les nouvelles, les médias tadjiks sont approvisionnés par les agences de presse officielles, en premier lieu, l'agence Tas, Interfax et par l'agence tadjik Khaver. Ce qui renforce leur dépendance vis à vis du pouvoir. Le poids du totalitarisme n'ayant pas été effacé par l'indépendance, les pratiques restent de fait immuables en dépit des changements de gouvernement.

8 La presse officielle est un véritable organe du pouvoir, totalement insérée dans l'appareil d'État. De ce fait, même durant le bref épisode du gouvernement de coalition, la presse officielle est restée fidèle au Parti Communiste. Son retour au pouvoir en automne 1992 a mis fin à l'existence d'une presse d'opposition, et les journalistes qui ont soutenu la coalition ont été renvoyés, arrêtés ou assassinés. Le principal journal officiel est Sedâ-ye Mardom (la voix du peuple). Porte-parole officiel et organe du parlement, il publie régulièrement les déclarations du président ou du premier ministre et, dans leur intégralité, les sessions du parlement.

9 Les autres quotidiens de Douchanbé comme Jomhuriyat se sont spécialisés dans la critique de l'opposition. Ces journaux ont toujours présenté les chefs de l'opposition comme "les traitres", "les responsables de la guerre civile, les agents des gouvernements étrangers". Les grands reportages quotidiens diffusent des nouvelles horribles d'actes de guerre, récits de véritables boucheries qui relatent par exemple, avec force détail, la façon dont "les hommes armés de l'opposition, sous la direction du grand chef spirituel Akbar Tourajânzâdeh, ont écorché des soldats pro-communistes ". Or, par son impact significatif sur la population, c'est en fait la radiotélévision qui est le principal moyen de la guerre médiatique et le principal enjeu de la guerre tout court. En 1992, la prise du bâtiment de la télévision par l'opposition sonne le glas du gouvernement communiste et annonce l'avènement du gouvernement de coalition. Depuis l'indépendance, la radio et la télévision ont changé trois fois de mains en même temps que le pouvoir. Les journalistes, qualifiés de "traîtres du peuple" ont fait l'objet de sinistres chasses aux sorcières.

11 Le contrôle des outils de communication étant évidemment essentiel pour la diffusion de la propagande à l'échelle nationale, les médias tadjiks sont devenus les principales caisses de résonance des luttes de pouvoir. Instruments du pouvoir et de revanche, la radio et la télévision ont transformé le Tadjikistan en un immense tribunal prenant le peuple en otage de la guerre des chaînes, et en définitive, les médias ont servi les mécanismes de la haine mis en oeuvre dans les enjeux de la guerre civile.

La mort précoce de la presse non officielle

12 Malgré la persistance de pratiques et de réflexes hérités de sept décennies de régime totalitaire, la presse tadjik a connu un vent de liberté. De juin 1991 - début de la Perestroïka tadjik - jusqu'au retour au pouvoir des communistes, en automne1992, le Tadjikistan a connu une floraison de journaux "libres". Plus d'une vingtaine de nouveaux titres sont apparus, de genres très divers, aussi bien littéraires que politiques.

13 Mais cette éclosion fut de courte durée car les journaux indépendants ont dû rapidement faire face à d'importantes difficultés, comme le prix élevé du papier qu'ils payaient au quintuple par rapport aux journaux officiels, ou encore pour trouver des 
imprimeries. Les imprimeries officielles n'acceptaient pas, bien entendu, de publier des journaux critiques à l'égard du gouvernement. Ce qui explique que très vite, seuls les journaux appuyés par des partis politiques aient pu se maintenir.

La presse d'opposition, quant à elle, a vécu plusieurs étapes caractéristiques depuis l'indépendance. Presse libre et pluraliste au départ, elle s'est très rapidement réduite à une presse d'opinion, le plus souvent sous le contrôle des partis politiques ou avec leur soutien.

La grande majorité des médias d'opposition, avant leur interdiction, étaient essentiellement des périodiques. Publiés depuis la capitale, ils fonctionnaient à partir de correspondants locaux opposés au Parti Communiste. Lieu de règlements de comptes politiques, ils s'opposaient avec virulence aux responsables gouvernementaux, les accusant de corruption, de malversations, critiquant leur politique locale ou nationale, dénonçant leur nostalgie de l'ère communiste.

Les trois partis principaux (le mouvement Rastâkhiz, le Parti Démocrate et celui de la Renaissance Islamique) publiaient chacun son journal, respectivement: Rastâkhiz (3000 exemplaires), Edâlat (entre 20000 et 30000 ) et enfin Nejat (entre 3000 et 5000 ). Ces faibles tirages et les difficultés de diffusion à l'échelle du pays témoigne du malaise de la presse écrite comme de l'absence de confiance du public vis à vis des journaux.

Avec (30 000) exemplaires, le principal journal post-indépendance était Tcherâq-e Rouz. Fondé par un groupe de journalistes critiques à l'égard du régime communiste il était proche, à l'origine, du parti islamo-démocrate, et fut baptisé symboliquement "lumière du jour". Son directeur, Dâdâjân Atta'ollâhi soulignait avec humour: "il y a eu tellement d'obscurité politique au Tadjikistan qu'on était même obligé de faire de la lumière pendant le jour".

18 Au dela des problèmes politiques, la presse subit aussi une crise d'ordre économique. Longtemps, le papier, monopole d'État, était importé de Russie. Aujourd'hui, avec l'inflation galopante, la faiblesse de la monnaie tadjik et les quotas d'importation, un kilo de papier de journaux invendus coûte aussi cher qu'un kilo de papier vierge. Les journalistes sont licenciés et les petits journaux indépendants ne peuvent survivre et ferment. D'autres, autrefois quotidiens, ne sont plus imprimés que deux ou trois fois par semaine. La crise est d'autant plus aigüe que la presse, totalement financée par l'État, ne dispose d'aucune recette publicitaire. Les journaux se vendent exclusivement en kiosque. Par ailleurs, les transports perturbés par la guerre civile et le prix de l'essence gênent la distribution de la presse sur le territoire.

La persécution des journalistes

19 Le journaliste au Tadjikistan jouissait d'un prestige important. Ces diplômés de Lettres ou d'école de journalisme étaient considérés comme d'éminents intellectuels. Aujourd'hui, après l'indépendance, la profession a perdu de son crédit. Moins bien rémunérés, les journalistes se doublent souvent de business-men.

En 1991, les collaborateurs des journaux appartenant aux partis politiques ont été les premiers acteurs de la guerre médiatique. Les intellectuels de la période postgorbatchevienne, universitaires, hommes de lettres devenus journalistes, ont commencé à utiliser leur plumes comme des armes contre l'idéologie communiste qui les avait opprimés pendant longtemps. L'heure des règlements de compte avait sonné ainsi qu'en témoigne la virulence des critiques à l'égard des chef du Parti Communiste. 
21 Les journalistes, portes-parole des partis d'opposition, se sont ainsi retrouvés propulsés à l'avant de la scène politique. Le parcours d'Ahmad Shâh Kâmel, le rédacteur en chef du journal Rastâkhiz est particulièrement significatif de cette évolution. Porte-parole de l'opposition pendant les 50 jours de manifestations anti-communistes à Douchanbé; il devint directeur de la télévision au moment où le gouvernement de coalition de l'opposition arriva au pouvoir; puis, comme plusieurs autres opposants, il fut poursuivi dès la montée en puissance des Communistes. Arrêté alors qu'il tentait de quitter le pays, jeté en prison, torturé, et détenu durant deux ans, il ne fut libéré qu'en décembre 1994, dans le cadre d'un échange de prisonniers (avec des soldats russes). Depuis, il est réfugié en Afghanistan.

Les rédacteurs de la presse indépendante furent également incarcérés ou tués lors du retour des communistes. Selon Salim Ayyoub "même pendant la période de la floraison des journaux, les journalistes étaient sous la surveillance du KGB. Ils recevaient souvent des menaces anonymes". Le matraquage public du directeur deTcherâq-e Rouz pendant les grandes manifestations du printemps 1992 illustre la violence et la confusion politique dont ils ont été victimes.

23 Ainsi, la restauration de l'ordre communiste a aggravé la situation des journalistes qui avaient cru pouvoir exercer leur métier librement. L'ancien conseiller du président Nabiev, et actuellement ministre du transport, Nazrellah Doustov, avait déclaré publiquement lors de la session du parlement tadjik en novembre dernier: "si nous arrivons au pouvoir, les premiers à être torturés seront les journalistes des journaux indépendants, car ils ont conduit les Tadjiks à s'opposer à Nabiev".

En effet, dès le lendemain de la reprise en main de Douchanbé par les communistes, le bâtiment principal de la presse fut encerclé, plusieurs bureaux et maisons d'administrateurs et de collaborateurs de presse furent pillés. C'était la fin de la liberté d'expression au Tadjikistan. Les journalistes "libres" ont pris le chemin de l'exil, certains reprenant leur publication dans la clandestinité. Ceux d'entre eux restant au Tadjikistan, ont continué à travailler sous le contrôle total du régime au pouvoir.

La presse parallèle

25 C'est dans les camps de réfugiés tadjiks, en Afghanistan qu'est apparue une nouvelle forme de presse, la plus simple qui soit et la plus spontanée: la presse murale. Les moyens sont rudimentaires, de grands cartons récupérés des stocks des produits alimentaires distribués dans les camps, accrochés sur les tentes du Haut Commissariat aux Réfugiés, les informations sont écrites à la main. On y trouve aussi bien des slogans vengeurs, des promesses de revanche, des insultes contre le gouvernement tadjik que des oeuvres poétiques sur le drame de l'exode, la tragédie de l'arrachement et la nostalgie de la terre natale. Cette forme de presse, réduite à sa plus simple expression, contribue à propager un climat de tension et de mobilisation auprès des populations les plus durement touchées par la guerre.

Contrairement à cette éclosion spontanée d'une forme de presse adaptée à la situation des camps de réfugiés, la presse d'opposition, décapitée et persécutée au Tadjikistan s'organise dans la clandestinité et dans l'exil.

27 Tcherâq-e Rouz, le journal le plus important de l'après indépendance, devient le principal journal de l'opposition. Désormais publié à Mosou, il se veut au-dessus des partis et revendique son indépendance. Son directeur insiste sur le fait que les journalistes qui travaillent n'ont pas le droit de militer dans un parti. Il est 
principalement financé par des hommes d'affaires tadjiks ou russes qui sont intéressés par l'avenir économique du Tadjikistan. Ainsi, Alexis Simonov, le très influent homme d'affaires russe qui finance plusieurs journaux des républiques d'Asie centrale contribue aussi au financement de Tcherâq-e Rouz.. Le tirage du journal qui était tombé à 10000 exemplaires depuis son installation à Moscou a presque doublé en dix mois. En Russie, l'opinion est divisée: une partie soutient le régime actuel, l'autre, démocrate, soutient les islamo-démocrates. Ce qui explique l'aide apportée par un homme d'affaire russe à un journal de l'opposition tadjik.

Tcherâq-e Rouz qui circule clandestinement au Tadjikistan, reste le journal le plus lu de la capitale. Il constitue une source importante d'informations, et ce même pour les autorités néo-communistes. Dans les ministères et les bureaux, les fonctionnaires parlent des articles publiés dans le journal. Lors des pourparlers pour les négociations de paix entre l'opposition et le gouvernement, les divers responsables politiques actuels du Tadjikistan n'hésitaient pas à faire allusion à l'entretien du chef spirituel tadjik, Qâzi Tourajânzâdeh, publié dans Tcherâq-e Rouz . Et pourtant, être en possession de ce journal lors d'un contrôle conduit à une arrestation immédiate.

Tcherâq-e Rouz est également diffusé dans les camps de réfugiés tadjiks au Pakistan et en Afghanistan. Un des derniers numéros était consacré à un entretien avec Gorbatchev et à ses opinions sur l'avenir de l'Asie centrale. Tcherâq-e Rouz traite de sujets variés: politique étrangère, problèmes des réfugiés tadjiks ou problèmes économiques et sa dépendance financière envers les démocrates russes est reconnue par ses responsables. La Radio : un outil efficace de propagande

Partout au Tadjikistan, dans chaque maison, dans chaque bureau, dans les lieux publics, un poste de radio est accroché au mur, diffusant les programmes de la seule station existante, la station officielle. Elle est l'instrument par excellence de la propagande gouvernementale. Personne n'y échappe. Les émissions commencent à cinq heures du matin pour ne s'arrêter qu'à une heure du matin. Ainsi, dès l'aube, les Tadjiks sont soumis à un incessant lavage de cerveaux qui dure jusqu'au soir, les soirées étant essentiellement consacrées à des émission musicales.

31 Le bâtiment de la radio est la cible privilégiée de chaque gouvernement arrivé au pouvoir. Lieu très surveillé par les groupes armés, il est aussi très protégé depuis la guerre civile. Celui qui détient la radio, contrôle aussi le pays. Situé en plein centre de la capitale, en face du parlement, le directeur de la radio siège au dernier étage. Il contrôle la totalité des programmes. Depuis la période soviétique, le fonctionnement de la radio n'a pas changé: la position du directeur de la télévision y est essentielle puisqu'elle donne aussi les commandes du ministère de la radio et de la télévision.

Informations et programmes sont contrôlés par le haut: le directeur règne sur toute l'information; les niveaux hiérarchiques inférieurs sont occupé par les chefs de service, puis les journalistes. Ces derniers composent avec une auto-censure incontournable. En ce qui concerne l'information générale, le chef de la radio reçoit, par téléphone ou courrier, les communiqués du gouvernement ou des informations qui doivent être diffusées. La majeure partie du temps, l'information est dictée par les responsables de presse des ministères qui rédigent eux-mêmes les communiqués et les envoient à la radio. La désinformation et la propagande sont particulièrement significatives au moment des flashes d'informations dont les plus importants et les plus longs sont à 7:00, 13:00 et 17:00. Ces informations sont l'occasion de critiques acerbes contre l'opposition. 
33 La radio essaye cependant de répondre aux besoins informationnels de la population, à majorité rurale, en diffusant de nombreux reportages sur les kholkhozes, l'agriculture, la production de coton, accompagnés de conseils pour les agriculteurs. Dans ces émissions on écoute souvent le témoignage direct des habitants des campagnes. Par ailleurs, de nombreux correspondants régionaux informent régulièrement, souvent par téléphone, les stations de radio sur ce qui se passent en province et dans les villages.

La musique, à laquelle la plupart des programmes laissent une grande part - musique ou interview avec les musiciens - rencontre un succès certain. Parmi d'autres émissions régulières de la radio les tables-rondes sur des thèmes qui préoccupent la population, ont aussi une audience importante.

Depuis l'exode de milliers de Tadjiks en Afghanistan et les déplacements à l'intérieur même du pays, la radio tadjik a adopté une nouvelle méthode de reportage servant à la propagande anti-opposition. Le programme qui dure du 5 à 7 heures du matin est consacré aux gens déplacés qui envoient des nouvelles aux membres de leur famille. Le journaliste de la radio fait une lecture déchirante de lettres, les nouvelles des proches étant largement agrémentées d'insultes contre les dissidents, d'accusations haineuses et d'appels à la revanche contre l'opposition, qui selon les présentateurs "a provoqué la guerre, a mis le pays à feu et à sang et est responsable des déchirures familiales".

Radio Tadjikistan a aussi un programme régional développé en persan, en dari, en ordou et en arabe. Ces émissions sont aussi accessibles dans tout le pays. L'émission en langue persane propose avec succès de la poésie déclaméees. Les informations provenant en totalité des agences officielles repésentent un moyen de propagande auprès des populations des pays voisins.

37 Parmi les radios étrangères reçues au Tadjikistan, les différentes chaînes de la radio russe sont les plus écoutées. Beaucoup d'ouzbekophones captent les radio d'Ouzbekistan. En outre, le Tadjikistan reçoit la BBC et radio Liberty en persan. La première a un impact très important et ses informations sont largement reprises dans les conversations particulières du lendemain. Radio France Internationale en persan reçoit une moindre audience car elle est difficilement reçue.

La radio irannienne, captée clandestinement pendant toute la période soviétique, a joué un rôle déterminant dans les évènements politiques au Tadjikistan. De nombreux slogans de la révolution iranienne ont ainsi été repris par les Tadjiks lors des manifestations du printemps 1992.

39 Au nord de l'Afghanistan, le gouvernement en exil dispose d'une radio basée dans le village de Tâlegân. Cette radio diffuse des émissions plusieurs heures par jour destinées aux camps de réfugiés mais qui atteignent aussi le territoire tadjik. Elle a pour politique essentielle de monter l'opinion publique tadjik contre le gouvernement en place à Douchanbé. La plupart des émissions est basée sur des reportages sur la situation des réfugiés et rappelle constamment à travers des témoignages, les atrocités et les massacres de l'armée pro-communiste lors de la guerre civile.

Une étonnante régionalisation de la télévision

40 La télévision passe pour avoir été le premier responsable du déclenchement de la guerre civile. Toute l'histoire aurait démarré à cause d'un clin d'oeil. Le chef de l'opposition, Qâzi Akbar Tourajânzâdeh, bête noire des communistes au parlement, a fait un clin d'oeil au public au cours d'une session du parlement diffusée en direct par la télévision. Ce clin d'oeil a été interprété comme une véritable provocation et les 
parlementaires communistes ont appelé le peuple à manifester dès le lendemain. Des milliers de personnes, encadrées par les chefs de kholkhozes, sont venues des quatre coins du pays pour manifester contre le "clin d'oeil de Qâzi".

41 L'impact de la télévision est tel que toute vie s'arrête dès qu'un programme important commence. Quelque soit l'heure de la journée, chacun lâche ce qu'il a dans les mains pour se retrouver devant le petit écran. Des postes sont disponibles dans toutes les maisons, même dans les villages, dans les bureaux des ministères ou des sociétés. L'essentiel des programmes est constitué des discours politiques, des interventions du président, des sessions parlementaires. Une série télévisée cubaine,"Les riches pleurent aussi", diffusée par la télévision de Moscou remporte un grand succès populaire.

Depuis l'indépendance, la télévision a vu son audience augmenter: elle est devenue le seul moyen de contact avec l'extérieur. Avant, la population fréquentait les restaurants, les cafés ou les réunions politiques, la vie sociale était intense. Aujourd'hui au coucher du soleil, la circulation étant limitée à cause de l'insécurité, la population reste chez elle devant la télévision, devenue l'unique distraction.

Il existe une seule chaîne officielle et centrale tadjik, diffusée sur tout le territoire. Ses programmes commencent au début de l'après-midi. Avant que le gouvernement ouzbek ne coupe les émissions, les Tadjiks d'Ouzbekistan pouvaient aussi regarder cette chaîne. Les programmes sont tous en langue tadjik, à l'exception d'une demi-heure d'informations en langue russe. Hormis les nombreuses heures de programmes musicaux, de films ou de séries téléviées tadjiks, on y voit beaucoup d'informations et de débats politiques. La télévision a été largement utilisée comme moyen d'endoctrinement par chacun des groupes successivement au pouvoir. Nous avons vu que l'ancien directeur de la télévison du temps des islamo-nationalistes, Ahmad Shâh Kâmel, n'a pas échappé au réglement de compte lors de l'arrivée des nouveaux dirigeants en1992.

Du côté des chaînes régionales, la région la plus développée, Khojend, au nord du Tadjikistan, dispose de sa propre télévision. Elle jouit d'une grande autonomie et peut même interrompre la chaîne officielle pour diffuser ses propres émissions. La télévision de Khojend diffuse beaucoup de musique, mais aussi des émissions et informations régionales, notamment celles relatives au développement économique locale. Il est vrai que depuis la guerre civile, le Khojend reste une région à part; il a connu un essor économique plus marqué que dans le sud du Tadjikistan. Les programmes de la télévision témoignent de cette avance, et la présence importante de la publicité reflète en particulier, la prospérité du nord du Tadjikistan. La télévision régionale de Khojend émet ses propres informations mais utilise aussi celles de la télévision centrale et celles en russe.

Dans la région de Kouliab, au sud du Tadjikistan, les groupes pro-communistes qui avaient perdu le pouvoir sur la capitale pendant la guerre civile en 1992 ont réussi à mettre en place une station de télévision privée. Après avoir coupé l'antenne centrale de la télévision du gouvernement, disposant seulement des moyens d'un petit studio, ils ont diffusé leurs propres émissions sur la région de Kouliab. On pouvait y voir les discours du chef armé pro-communiste, Sangak Safarov, des vidéos tournées pendant les combats de village entre islamo-démocrates et communistes et les prisonniers de guerre. Sangak Safarov apparût pour la première fois à la télévision officielle au cours d'une interview en octobre 92, alors qu'il passait fréquemment sur la télévision privée 
de Kouliab pendant les six mois précédents. Arrivés au pouvoir, les néo-communistes mirent fin aux activités de cette chaîne, par essence média de résistance .

La télévision privée représente un phénomène nouveau et il n'existe encore qu'une seule chaîne de télévision privée au Tadjikistan:Teymour Malik. Elle se situe dans la région de Khojend et appartient au gouverneur de cette région. Fonctionnant sur ses recettes publicitaires, cette chaîne commerciale passe essentiellement des films indiens, auquels elle doit sa popularité, entrecoupés de spots publicitaires. Seule la région du nord en bénéficie, entre 19 et 24 heures.

Parmi les chaînes étrangères, les plus regardées restent les chaînes russes et notamment celle destinée plus particulièrement aux républiques de l'ex-Union Soviétique, à savoir la chaîne Ostankino. Très regardée au Tadjikistan, elle fournit des informations majeures sur les événements en ex-Union Soviétique. Les Tadjiks la préférent aussi pour ses séries télévisées et ses films américains, en particulier les films de karaté.

La chaîne ouzbek vient ensuite, facilement captée sur tout le territoire, elle est regardée non seulement par les $23 \%$ d'Ouzbek qui y vivent mais aussi par les Tadjiks. Les émissions de la chaîne turque destinées à l'Asie centrale, très populaires, ne peuvent pas être facilement captées au Tadjikistan, faute d'émetteur, mais les Ouzbeks de ce pays peuvent voir certaines programmes de cette chaîne via les chaînes ouzbeks. La télévision iranienne, entre le 19 septembre 1991 et l'arrivée au pouvoir du nouveau régime en novembre 1992, émettait un programme hebdomadaire d'informations générales en persan pour tous les pays d'Asie centrale. Ouvertes par des chants révolutionnaires, ces informations étaient présentées par des femmes voilées, ce qui n'a pas manqué d'étonner les Tadjiks. Les émissions de la télévision iranienne furent cependant interrompues par le gouvernement en novembre 1992, en raison de ses sentiments hostiles aux iraniens.

51 Les ambassades et les organisations humanitaires restent les seules à disposer des antennes paraboliques qui suppléent aux carences du téléphone et à la rareté des journaux étrangers. La télévision satellite jouent donc un rôle limité. En revanche,les cassettes audio et vidéo ont un réel marché au Tadjikistan, des films indiens circulent partout et les enregistrements de variétés venant des pays voisins et de l'Occident remportent un grand succès aupès de la population.

53 L'euphorie de la liberté d'expression au Tadjikistan n'aura duré qu'un temps avant de tourner à la récupération politique. La presse est rapidement devenue l'instrument des partis, l'enjeu et l'arme de leur lutte pour le pouvoir et pour le contrôle de l'opinion publique tadjik. De ce fait les journalistes, partie prenante des appareils politiques et acteurs de premier plan, ont été les principaux protagonistes de la guerre médiatique qui a contribué à faire monter les tensions au Tadjikistan. Peu soucieuse d'information ou d'objectivité, la presse tadjik, officielle ou d'opposition, contribue par sa propagande à entretenir la haine, garder ouvertes les blessures d'hier et préparer les combats de demain.

Avâz-e tadjk : un journal persanophone de l'Ouzbekistan

Le Tadjikistan, avec 6 millions d'habitants, est le seul pays persanophone de l'Asie centrale. Mais un nombre important de Tadjiks (selon les chiffres officiels un million et selon des sources non-officielles huit millions) habitent dans les régions de Samarcand 
et Boukhara en Ouzbékistan. Les Tadjiks de l'Ouzbékistan ont une presse locale, dont le plus important est Avâzé-tadjik.

Ayant quatre pages, publiés deux fois par semaine, ce journal a un tirage de 7000 exemplaires actuellement, contre 10000 l'an dernier. Sa une est consacrée aux informations politiques de l'Ouzbékistan et la dernière est réservé aux programmes de la télévision. Seulement deux pages centrales traitent de la vie des Tadjiks.

Ce journal a eu 70 ans en 1994. Créé par Sadr Edin Ayni, le grand poète et homme de lettres tadjik, il s'appelait au départ Avâz-e tadjik-e kam baqale (la pauvre voix du peuple) et à son dixième numéro, changea son nom en Avâz-e tadjik (la voix des tadjiks). En 1931, le journal est rebaptisé Haqiqat-e Ouzbékistan (la réalité de l'Ouzbékistan), puis sa publication est interrompue pendant la Seconde guerre mondiale et jusqu'en 1950. Il réapparaît sous le nom d' Ouzbékistan-e Sorkh (L'Ouzbékistan rouge) jusqu'en 1964 et s'intitule ensuite "Réalité de l'Ouzbékistan" avant de redevenir, en 1992, "La voix du tadjik".

57 Aujourd'hui il est une branche du journal du parlement, le quotidien Khalq Souzi (la voix du peuple). La traduction de ses informations officielles est aussi lue dans le journal du parlement en langue ouzbek, kazakh ou russe.

A titre d'exemple lisons l'édition du 11 février 1995. La une rapporte les paroles du président Karimov au cabinet du ministre, les félicitations des chefs d'État arabes au président Karimov pour le mois de Ramadan, des informations sur l'augmentation des salaires. Dans les pages centrales, on lit un article qui développe certains aspects de la culture tadjik, la vie de Fârâbi le philosophe iranien, et des informations locales. Il y a aussi un long entretien avec l'ambassadeur d'Iran en Ouzbékistan dans ce numéro. 